\title{
Probing the uniformity of silver-doped epitaxial graphene by micro-Raman mapping
}

Ivan Shtepliuk, Ivan Gueorguiev Ivanov, Nikolaos Pliatsikas, Tihomir Iakimov, Andreas Jamnig, Kostas Sarakinos and Rositsa Yakimova

The self-archived postprint version of this journal article is available at Linköping University Institutional Repository (DiVA):

http://urn.kb.se/resolve?urn=urn:nbn:se:liu:diva-163649

N.B.: When citing this work, cite the original publication.

Shtepliuk, I., Ivanov, I. G., Pliatsikas, N., Iakimov, T., Jamnig, A., Sarakinos, K., Yakimova, R., (2020), Probing the uniformity of silver-doped epitaxial graphene by micro-Raman mapping, Physica. B, Condensed matter, 580, 411751. https://doi.org/10.1016/j.physb.2019.411751

Original publication available at:

https://doi.org/10.1016/j.physb.2019.411751

Copyright: Elsevier

http://www.elsevier.com/

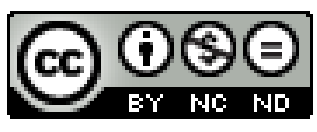




\title{
Probing the uniformity of silver-doped epitaxial graphene by micro-Raman mapping
}

\author{
I. Shtepliuk ${ }^{* 1,2}$, I. G. Ivanov ${ }^{1}$, N. Pliatsikas ${ }^{1}$, T. Iakimov ${ }^{1}$, A. Jamnig ${ }^{1}$, K. Sarakinos ${ }^{1}$, and \\ R. Yakimova ${ }^{1}$ \\ ${ }^{1}$ Department of Physics, Chemistry and Biology, Linköping University, SE-58183, \\ Linköping, Sweden \\ ${ }^{2}$ Frantsevich Institute for Problems of Materials Science, NASU-National Academy of \\ Sciences of Ukraine, 142 Kyiv, Ukraine
}

\begin{abstract}
We present a Raman spectroscopy study on epitaxial graphene decorated with thin Ag films (2$15 \mathrm{~nm}$ ), which are deposited using magnetron sputtering. We find that the presence of $\mathrm{Ag}$ on the graphene surface induces doping, the uniformity and efficiency of which is determined by Ag nominal thickness. Deposition of Ag films with thicknesses up to $5 \mathrm{~nm}$ favors the effective electron transfer from Ag to epitaxial graphene. A significant redshift and broadening of the $2 D$ peak are observed with increasing the Ag-layer thickness above $5 \mathrm{~nm}$, which is indicative of large strain and doping fluctuations. We also observe a non-trivial linear growth of $2 D / G$ peak intensity ratio with increasing $D / G$ ratio for all $\mathrm{Ag}$-decorated samples, which is explained by increase of peak amplitude due to surface enhanced Raman scattering and charged impurityinduced screening caused by the presence of $\mathrm{Ag}$ on the graphene surface.
\end{abstract}

Keywords: epitaxial graphene, silver, Raman mapping, doping, strain

*Corresponding author: ivan.shtepliuk@liu.se

\section{Introduction}

As a unique member of the graphene family, epitaxial graphene grown on different polytypes of $\mathrm{SiC}(\mathrm{EG} / \mathrm{SiC})$ possesses a wide range of intriguing properties, including large area, high quality, low defectiveness, chemical and thermal stability, and transfer-free growth [1-5], thereby triggering an enormous interest within scientific community during last 15 years and provoking researches that yielded a more subtle understanding how to exploit this material in real applications, especially in electronics [6, 7], optics [8] and sensorics [9-12]. It is obvious that the development of next-generation devices based on $\mathrm{EG} / \mathrm{SiC}$ requires its incorporation/integration into/with classical semiconductor technologies ( $p-n$ junctions, transistors, Schottky contacts etc.) and achieving uniform doping over prescribed nano-scale 
regions. These interrelated tasks are still very challenging due to poor reproducibility of the doping methods (substitutional doping or electron charge transfer) that usually cause the significant reduction of the electrical performance of epitaxial graphene and its structural and morphological damage. Eventually, the trade-off in the framework of triad "doping efficiency - EG conductivity - structural homogeneity" must be reached to push graphene closer to high performance devices. In this regard, any attempts to bring new insights into doping mechanisms of graphene toward reliable manipulation of its electronic properties on centimeter-scale are of high scientific and technological relevance. In this study, we are focusing on investigating the doping phenomena induced by deposition of thin silver (Ag) films on large-area epitaxial monolayer graphene.

The choice of Ag as a model metal to dope graphene is justified by its unique properties with respect to graphene [13]. It is known that the deposition of Ag nanoparticles on graphene may produce $n$-type doping driven by the work function difference between the two materials [14]. This is because the Fermi level alignment makes it easier for charge carriers to move from the Ag to graphene, thereby lifting Fermi level of graphene above the Dirac point. Furthermore, according to earlier density functional theory (DFT) calculations, Ag interacts with graphene mainly through van der Waals forces enabling formation of ultra-thin Ag films weakly bonded to substrate [15]. Such features combined together provide excellent prerequisites for fabrication of damage-free large-area uniformly $n$-type doped epitaxial graphene, since low defect density and pronounced electron charge transfer at the interface are expected as a result of interaction between defect-free graphene structure and Ag.

Despite the large technological potential of $\mathrm{Ag} / \mathrm{EG} / \mathrm{SiC}$ nanoarchitectures, only few reports in the literature have studied the effect of Ag directly interfacing with epitaxial graphene [16-22]. Earlier studies on the formation of Ag films on EG/SiC employed high-vacuum thermal evaporation [20] and Ag deposition from chemical solution [17]. Sekine et al. [20] reported a synthesis of highly crystalline $\mathrm{Ag}$ nanosized islands with a face-centered cubic structure and a distance to epitaxial graphene of $\sim 3.14 \AA$, pointing out the physiosorbed state without formation of chemical bonding. It was also found that the decoration of epitaxial graphene with Ag causes increase of the graphene electron density from $1 \times 10^{12} \mathrm{~cm}^{-2}$ (pristine graphene) to $1.9 \times 10^{13} \mathrm{~cm}^{-2}$. Chadhari et al. [17] reported the formation of pyramidical-shaped silver islands with average diameter of $18 \mathrm{~nm}$. There have been also several attempts to grow ultra-thin films by evaporation from a water-cooled Knudsen cell onto EG/SiC samples with different thicknesses at a substrate temperature of $77 \mathrm{~K}[19,21]$. Adjusting the thermal decomposition temperature of 6H-SiC Liu et al. [19] controlled the thickness of graphene prior 
to Ag deposition, thereby providing different templates (from "pure" buffer layer to few-layer graphene) for synthesis of the metal nanoparticles. It was claimed that Ag-island size during the initial film growth stages is virtually insensitive to the thickness of epitaxial graphene, while increase of the graphene layer thickness causes reduction of $n$-type doping due to the weakened interaction between Ag islands and thicker EG layers. In their more recent work [21], the same group showed substantial evidence of substitutional doping of EG with Ag, and Ag intercalation via penetration through edge and defect sites in the topmost graphene layers. In this case, $\mathrm{Ag}$ atoms preferentially form covalent bonds with carbon atoms. Similar chemical interaction phenomena occurring at the interface between liquid silver and graphene-coated SiC substrate were independently examined by Homa et al. [18] by using sessile drop method. Moreover, an enhanced Ag island nucleation density was reported when depositing on the buffer layer (carbon rich surface reconstruction of the $4 \mathrm{H}-\mathrm{SiC}$ ) [21].

The findings highlighted in the previous paragraph suggest that the quality of graphene plays an important role for the Ag-layer nucleation and growth. A high number of intrinsic defects and edge states in graphene may significantly enhance the adsorption ability of $\mathrm{Ag}$ on graphene and hence yield a more irregular Ag/graphene interface and/or Ag-C intermixing. Thus, only high-quality defect-free graphene could ideally satisfy the requirements of effective n-type doping during deposition of Ag films. In the light of that, epitaxial monolayer graphene with high thickness uniformity is regarded as promising template for deposition of electrondonating Ag-layers. In this context, achieving control over both the graphene uniformity after $\mathrm{Ag}$ deposition and Ag-induced doping efficiency is an important precondition for further progress in conceptualization of the EG-based devices.

Micro-Raman mapping analysis is an excellent approach to elucidate the nature of structural changes in epitaxial graphene upon $\mathrm{Ag}$ deposition and to draw conclusions with respect to doping uniformity and mechanisms. This is because the intrinsic Raman modes (namely $G$ and $2 D$ ) of epitaxial graphene are strain- and doping-depended [23, 24], then any variations in the position and intensity of these peaks enable to gauge strain/doping changes due to $\mathrm{Ag}$ presence on the substrate surface. Furthermore, statistical analysis of Raman data [25-28] can be used to estimate the degree of spatial fluctuations of the carrier concentration in the Ag-coated EG/SiC. This route has not been explored in the literature so far.

In the present paper, we investigate the formation of thin Ag films deposited on epitaxial graphene/SiC by magnetron sputtering and study their effect on the vibrational properties of epitaxial graphene. A deep understanding of carrier density modulation induced by $\mathrm{Ag}$ 
deposition is of special interest because it provides critical knowledge toward optimization of the graphene doping conditions.

\section{Experimental procedures}

Thin Ag films were deposited by direct current (dc) magnetron sputtering in an ultra-high vacuum chamber (base pressure $\sim 10^{-8} \mathrm{~Pa}$ ) at room temperature. Ar gas (purity 99.999\%) at a pressure of $1.7 \mathrm{~Pa}$ was used to generate plasma and sputter a magnetron source which was equipped with an Ag target (diameter $76.2 \mathrm{~mm}$; thickness $6 \mathrm{~mm}$; purity 99.99\%). The nominal thickness of the Ag films was varied between 2-15 $\mathrm{nm}$ by changing the deposition time. As substrates for growth, we used $7 \mathrm{~mm} \times 7 \mathrm{~mm}$ on-axis $4 \mathrm{H}-\mathrm{SiC}(0001)$ wafers covered by monolayer graphene. Prior to deposition, the substrates were cleaned with ethanol followed by $\mathrm{N}_{2}$ drying.

Epitaxial graphene was synthesized by high-temperature thermal decomposition of the $\mathrm{Si}$-face $\mathrm{SiC}$ in argon atmosphere. Additional technical details on this sublimation technique can be found elsewhere [29]. The formation of monolayer graphene was confirmed by optical reflectance mapping (not shown here) [30] and subsequent Raman scattering studies.

The Ag-layer surface morphology was investigated using plan-view scanning electron microscopy (SEM) in a Leo 1550 Gemini SEM instrument at an operating voltage ranging from $10 \mathrm{kV}$ to $20 \mathrm{kV}$ and a standard aperture value of $30 \mathrm{~mm}$. The film crystal structure was studied by X-ray diffractometry (XRD) Bragg-Brentano geometry, using a Panalytical X'pert Pro instrument with $\mathrm{Cu} \mathrm{K} \alpha$ radiation $(\lambda=0.1542 \mathrm{~nm})$. XRD patterns (not shown here) show that $\mathrm{Ag}$ films have a fcc crystal structure, they are polycrystalline, and grown preferably along the [111] crystallographic direction. Raman mapping analysis was performed by using a micro-Raman setup based on a monochromator (Jobin-Yvon, model HR460) equipped with a CCD (couplecharged device) camera. The objective lens has a magnification of 100×and numerical aperture $\mathrm{NA}=0.95$ resulting in a $\sim 0.85 \mu \mathrm{m}$ diameter of the laser spot focused on the sample surface. $\mathrm{A}$ $532 \mathrm{~nm}$ diode-pumped solid-state laser with $1 \mathrm{~mW}$ power was used as an excitation source. The spectral resolution of the system is $\sim 5.5 \mathrm{~cm}^{-1}$. 121 Raman spectra on a square map ( $3 \mu \mathrm{m} \times$ $3 \mu \mathrm{m}, 0.3 \mu \mathrm{m}$ pitch) were collected to construct two-dimensional micro-Raman maps.

\section{Results and discussion}

Figure 1 displays the plan-view SEM images of Ag films deposited on EG/SiC substrates for nominal thicknesses ranging from 2 to $15 \mathrm{~nm}$. The surface of the $2 \mathrm{~nm}$ film (Fig. 1(a)) consists of isolated circular islands. Analysis of multiple images corresponding to the same nominal thickness showed that the mean island size is $17.6 \mathrm{~nm}$ with standard deviation of 5.3. Increase of the nominal film thickness to $5 \mathrm{~nm}$ (Fig. 1(b)) yields elongated islands with mean 
size of $42.3 \mathrm{~nm}$ with standard deviation of 15.9. Further deposition of Ag results in increase of island size which form an interconnected island network (10 nm in Figs 1(c)) which eventually leads to the formation a nearly continuous layer $(15 \mathrm{~nm}$; Fig. 1(d)). The morphological evolution observed in Fig. 1 is consistent with results for noble metal film growth on a variety of weakly-interacting substrates, including $\mathrm{SiO}_{2}$ and graphite [31, 32], and it is the result of the dynamic competition between island nucleation, growth, and coalescence [33].
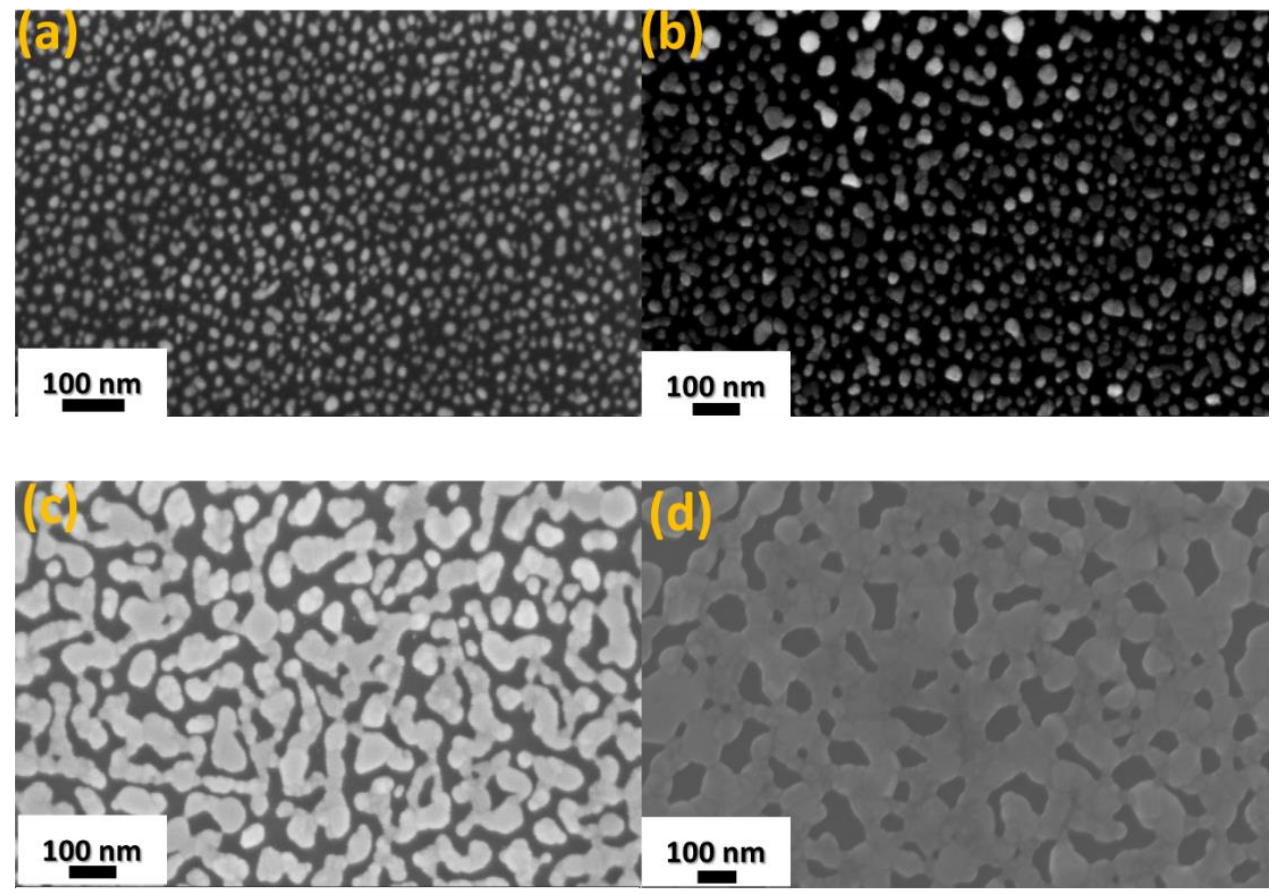

Figure 1. Plan-view SEM images of Ag films grown by magnetron sputtering on EG/SiC substrates at various nominal thicknesses: (a) $2 \mathrm{~nm}$; (b) $5 \mathrm{~nm}$; (c) $10 \mathrm{~nm}$; and (d) $15 \mathrm{~nm}$.

In order to establish the effect of Ag-layer morphology on the vibrational and structural properties of graphene we performed Raman spectroscopy measurements. Figure 2 shows the representative Raman spectra of the pristine and Ag-decorated (nominal thickness 2 to $15 \mathrm{~nm}$ ) epitaxial graphene. The spectrum of the pristine epitaxial graphene sample is dominated by two strong peaks at $1611 \mathrm{~cm}^{-1}$ and $2733 \mathrm{~cm}^{-1}$, which are attributed to characteristic $G$ and $2 D$ Raman in-plane vibrational modes, corresponding to atomic displacements of $s p^{2}$-bonded carbon species in graphene lattice [34]. We also notice the presence of the low-frequency scattering from the left side of $G$ peak ( $1200 \mathrm{~cm}^{-1}-1600 \mathrm{~cm}^{-1}$ spectral region), which is related to the buffer layer (interfacial C-rich layer between graphene and $4 \mathrm{H}-\mathrm{SiC}$, in which $30 \% \mathrm{C}$ atoms are covalently bonded to Si atoms) [35]. Defect-related vibrational modes are absent or very weak 
compared to the two main epitaxial graphene Raman peaks, suggesting the high-quality (close to defect-free) of the monolayer graphene before Ag deposition.

The decoration of the epitaxial graphene with Ag leads to the following changes in the recorded Raman spectra: (i) activation of intervalley scattering from $K$ to $K^{\prime}$ ( $D$ peak), intravalley scattering from $K$ to $K\left(D^{\prime}\right.$ peak) and combination scattering $(D+G$ peak) $[36,37]$ (see Figure 2 and inset of Fig. 2), which is indicative of generation of in-plane and out-of-plane defects; (ii) red shift and broadening of $G$ and $2 D$ modes, which may originate from synergetic influence of both strain and doping [38]; and (iii) enhancement of Raman signal, which can be ascribed to a combination of surface-enhanced Raman scattering (SERS) phenomena induced by both pronounced charge transfer at the interface $\mathrm{Ag} / \mathrm{graphene}$ interface and plasmonicallyactive Ag nanoparticles [39, 40]. Moreover, Fig. 2 shows a continuous shift from $1611 \mathrm{~cm}^{-1}$ to $1590 \mathrm{~cm}^{-1}$ and split of the $G$ peak with increasing Ag-layer thickness. The frequency shift is more pronounced for $2 D$ mode, for which the peak shifts from $2705 \mathrm{~cm}^{-1}$ (for epitaxial graphene decorated with $2 \mathrm{~nm} \mathrm{Ag} \mathrm{film)} \mathrm{to} 2689 \mathrm{~cm}^{-1}$ (for epitaxial graphene decorated with $15 \mathrm{~nm} \mathrm{Ag}$ film).

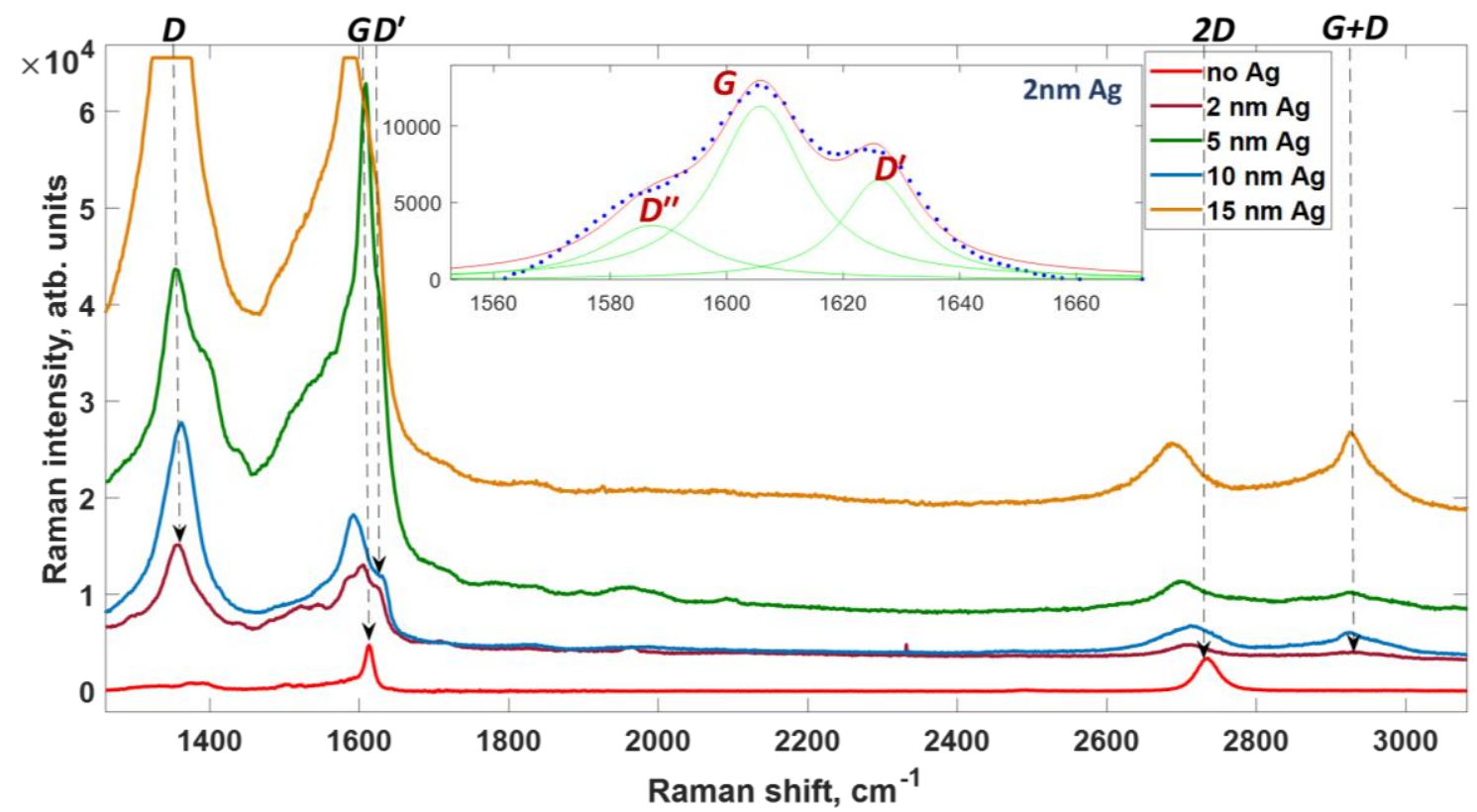

Figure 2. Representative Raman spectra of epitaxial graphene before and after deposition of Ag films with different thicknesses. Inset demonstrates the deconvoluted spectral region in the proximity of the $G$ peak, suggesting the $G$ peak splitting as a result of Ag decoration (nominal thickness $2 \mathrm{~nm}$ ).

Figure 3 presents a plot of the $2 D$ vs. $G$ peak position for all recorded spectra, for pristine graphene and $\mathrm{Ag}$-decorated $\mathrm{EG} / \mathrm{SiC}$ substrates at various nominal thicknesses, with the aim to 
decouple the effects of strain and doping on characteristic Raman scattering modes [41, 42]. The data points for pristine graphene sample before Ag deposition scatter mostly along a line with a slope of about 2.3 (blue circles in Fig. 3). This group of points is parallel to so-called strain line that agrees well with the literature data for purely stressed graphene subjected to biaxial compressive strain (due to the substrate effect) with negligible doping effect [43].

Compared to pristine graphene, there is no linear dependency seen for substrates interfacing with ultra-thin $\mathrm{Ag}$ deposits $(2 \mathrm{~nm}$ and $5 \mathrm{~nm}$; purple diamonds and green squares respectively) and the spread of the data points takes place in very narrow region. This behavior implies uniform doping over the scanned area of the Ag-decorated epitaxial graphene and is consistent with electron doping regime, for which the slope of $2 D / G$ line is negative $(\sim-0.1)$ [44].

The data points for epitaxial graphene decorated with $10 \mathrm{~nm}$ thick Ag film (red asterisks in Fig. 3) are neatly arranged along a strain line (from the left side), suggesting screening effect [45]. From this analysis it appears that $10 \mathrm{~nm} \mathrm{Ag}$ film is not suitable for uniform doping, since large non-uniform compressive strain occurs possibly due to coalescing process. During this process, the interconnections between initially separated Ag nano-islands start to appear, thereby favoring local strains and screening effects. On the other hand, $15 \mathrm{~nm}$ thick Ag film is more continuous in nature and, according to Figure 3 (violet triangles), is partly relaxed (since the corresponding data points scatter primarily along a line with the small negative slope), indicating nearly the same strain degree, but large carrier density fluctuations. It is most likely that compressive strain on graphene induced by the substrate effect from one side and $\mathrm{Ag}$ deposition at the early stages from another side can be released by structural transformation from the nano-island film to continuous silver layer.

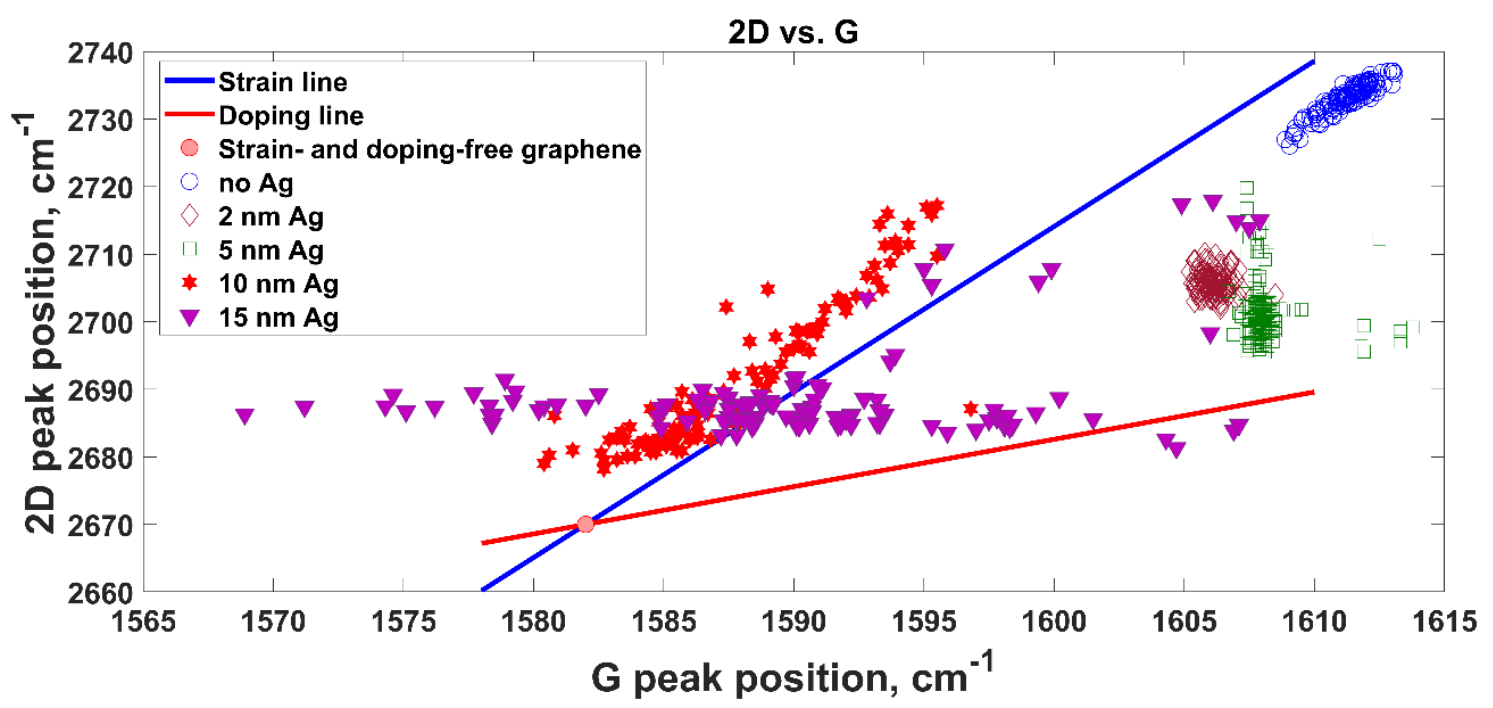


Figure 3. Mutual dependence of $2 D$ peak frequency and $G$ peak frequency for pristine and Ag-decorated epitaxial graphene obtained from two-dimensional Raman mapping. The light red point $\left(G=1582 \mathrm{~cm}^{-1}\right.$ and $\left.2 D=2670 \mathrm{~cm}^{-1}\right)$ denotes the strain- and doping-free graphene. Theoretical dependences of the $2 D$ peak position on $G$ peak position for graphene subjected only to strain or hole doping are also plotted for reference (blue and red lines, respectively).

To further support the conclusions from the results presented in Fig. 3, we then consider the evolution of FWHM of $2 D$ peak with Ag-layer thickness (Figure 4). Since this parameter is very sensitive to doping and structural quality of the graphene [41, 45], it can be also used to probe the uniformity of electronic properties of Ag-decorated epitaxial graphene. We observed dramatic changes of the width of the $2 D$ peak with $\mathrm{Ag}$ nano-film thickness increasing (see $2 \mathrm{D}$ frequency versus $G$ frequency dependences color coded by $\Gamma_{2 D}$, which are shown in Figure 4). To be more exact, the extracted values of FWHM of 2D peak are 33.3, 49.3, 44.54, 77.8 and $64.1 \mathrm{~cm}^{-1}$ (the corresponding histograms of $\Gamma_{2 D}$ distribution are shown in Fig. $4 \mathrm{f}$ ). Such band broadening can be explained by large strain fluctuations [45] and doping-induced phenomena [41], also indicating that only extremely thin silver films (2 and $5 \mathrm{~nm}$, respectively) may produce, to some extent, uniform $n$-doping.

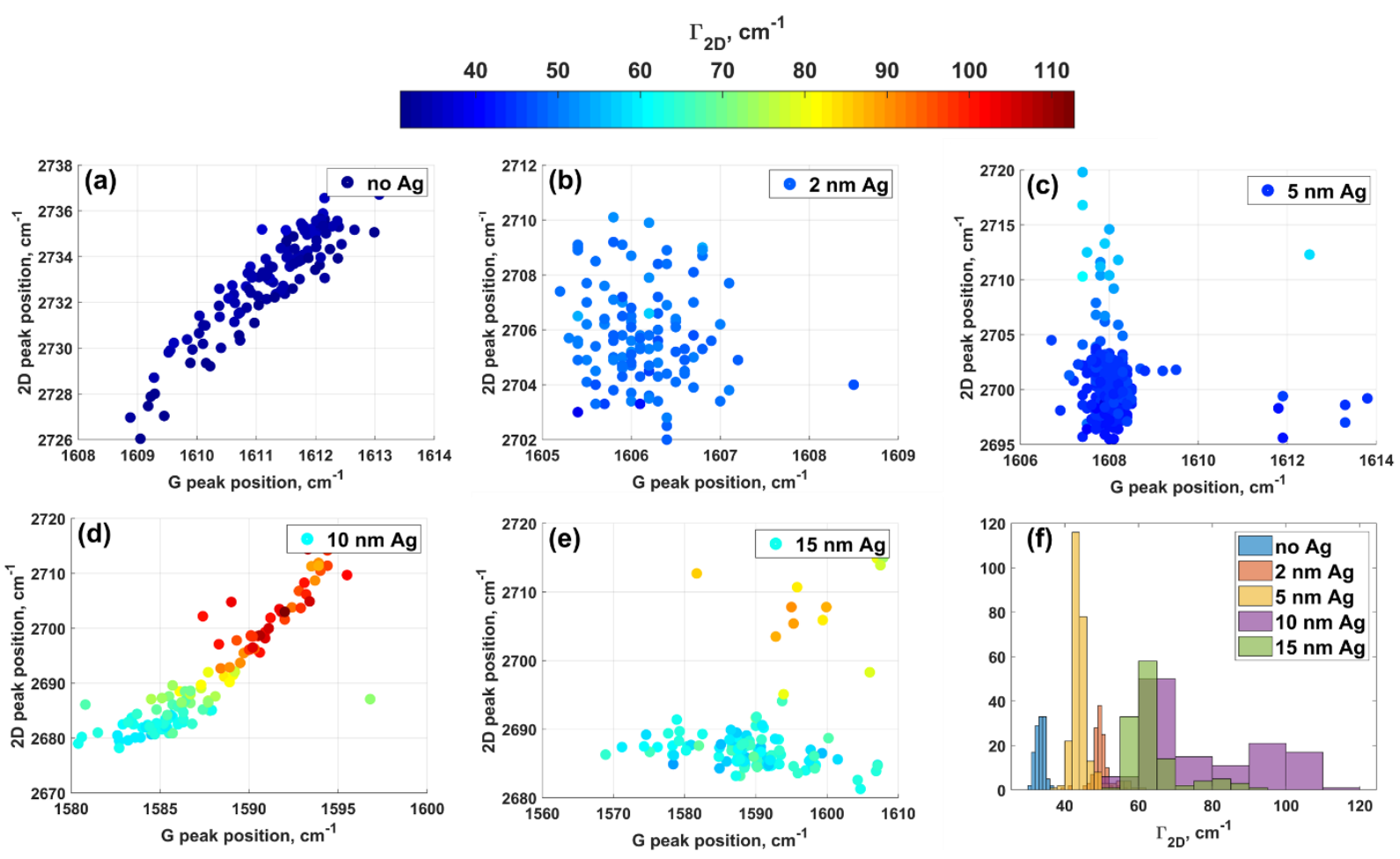

Figure 4. (a-e) Dependency of the $2 D$ peak position on the $G$ peak position for pristine and Ag-decorated epitaxial graphene color-coded by FWHM (full width at half maximum, $\Gamma_{2 \mathrm{D}}$ ) of $2 D$ peak. (f) Histograms of $\Gamma_{2 D}$ values for pristine and Ag-decorated epitaxial graphene. 
The variation in the $2 D / G$ amplitude ratio versus $G$ peak position shows also striking contrasts between the electronic properties of the epitaxial graphene before and after silver decoration (Figure 5a). Particularly, we revealed a weak dependence of the $2 D / G$ ratio on $G$ mode frequency and overall decrease in amplitude from 0.75 (for pristine graphene) to 0.1 and 0.06 for graphene affected by $2 \mathrm{~nm}$ and $5 \mathrm{~nm} \mathrm{Ag}$ layers, respectively. This finding provides additional evidence to support the claim on $n$-type doping of graphene [24] that is in good agreement with previously reported data on Ag-induced $n$-doping in exfoliated graphene [4648]. Unlike to effect of the ultra-thin Ag films on properties of epitaxial graphene, deposition of thicker films (10 and $15 \mathrm{~nm}$ ) leads to stronger dependence of the $2 D / G$ ratio on $G$ peak position and more random spread of data points, suggesting the large strain and doping fluctuations.

To gain more insights into the nature of Ag effect on epitaxial graphene, we also plotted the dependence of the $2 D / G$ amplitude ratio on $D / G$ amplitude ratio (Figure $5 \mathrm{~b}$ ). The linear behavior of such dependences is supposed to be related to the influence of Ag-induced defects. Nevertheless, these results contradict literature data, according to which the intensity of the $2 D$ line monotonously decreases with increasing the defect concentration [49]. We suggest that the observed linear dependences can be explained by: (i) different degrees of the enhancement (due to SERS effect) of $D$ and $2 D$ peak amplitudes, depending on the resonance frequency or energetics of the charge transfer channel; and/or (ii) local charged impurity-induced screening of the graphene $\pi$ electrons, followed by reduction of electron density and subsequent increase in $2 D / G$ ratio [24].
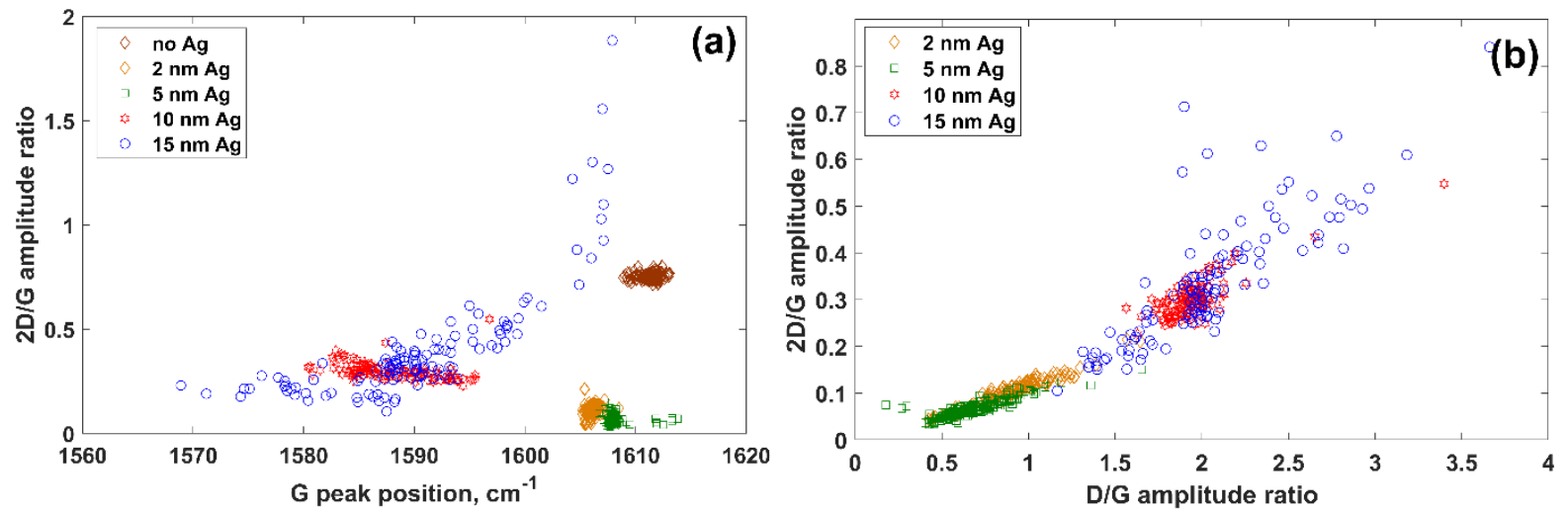

Figure 5. (a) $2 D / G$ amplitude ratio plotted as a function of the Raman shift of the $G$ mode pristine and Ag-decorated epitaxial graphene samples. (b) Correlation between $2 D / G$ amplitude ratio and $D / G$ amplitude ratio for Ag-decorated epitaxial graphene on $4 \mathrm{H}-\mathrm{SiC}$ 
By ascribing the carrier concentration and compressive strain values to each of the data points shown in Figure 3 for pristine epitaxial graphene and samples affected by the deposition of thin Ag silver films with thickness 2 and $5 \mathrm{~nm}$ (this approach is well documented in literature and successfully approbation for doped graphene under biaxial strain [24, 42, 44, 50, 51]), we constructed the carrier (electron) concentration maps (Figure 6) and strain distribution maps (Figure 7) with corresponding histograms,

The results in Figure 6 reveal that large area and quite uniform $n$-type doping is achieved as a result of $\mathrm{Ag}$ deposition. An increase in film thickness and area of coverage results in increase in doping level and an increase in spatial fluctuations of carrier concentration.

Figure 7 shows an overall decrease in compressive strain. Furthermore, local strain variations correlated with electron density distribution over the map regions of enhanced carrier density correspond to regions of reduced compressive strain. Such a correlation can be interpreted in terms of fundamental relationship between carrier concentration and strain proposed by Schmidt et al. [52]: an increase in the compressive strain gives rise to the decrease of in-plane length of C-C $s p^{2}$-bond and, consequently, the increase in Fermi velocity. Due to this reason, the electrons density becomes smaller. Therefore, biaxial compressive strain in epitaxial graphene after Ag deposition can be partly released by enhancement of the local electron concentration due to produced $n$-doping.

(a)
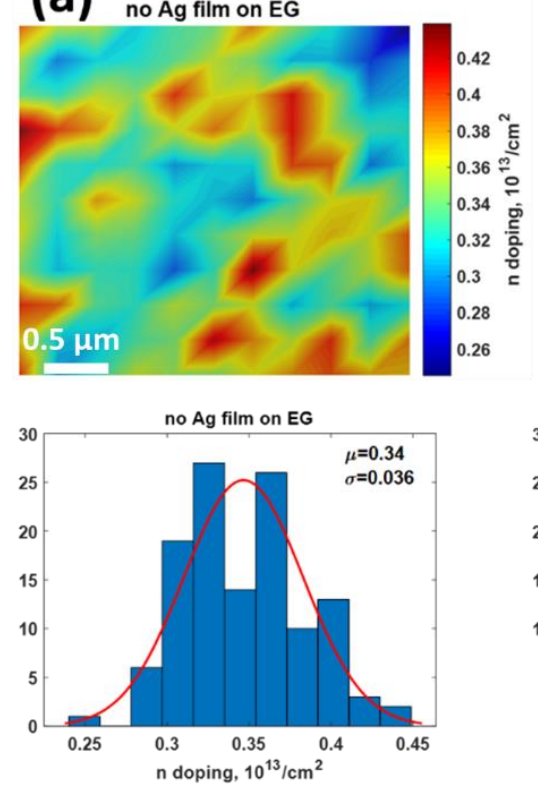

(b)
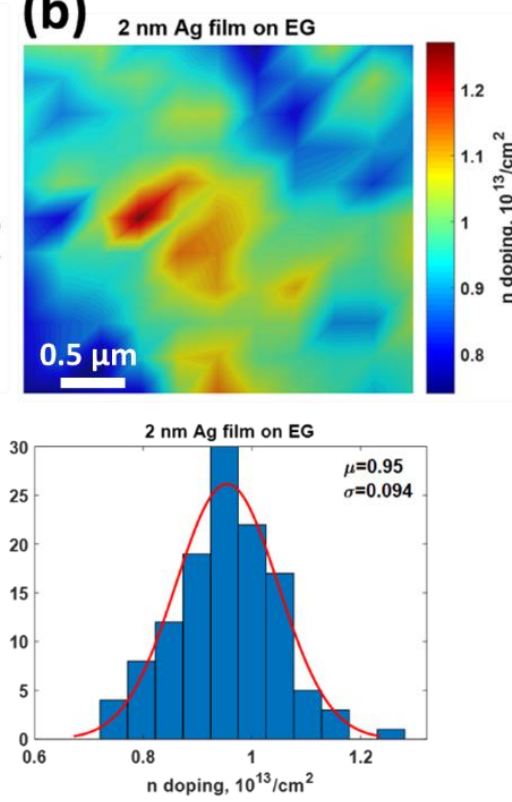

(c) $5 \mathrm{~nm}$ Ag film on $\mathrm{EG}$
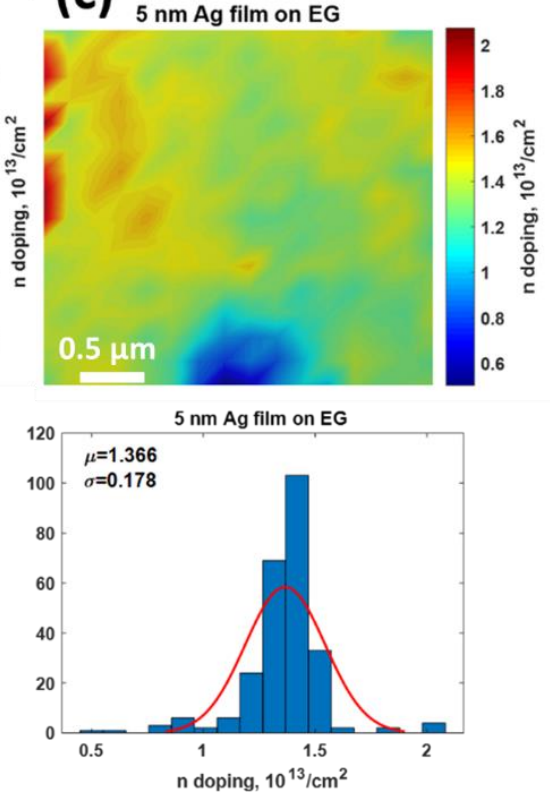

Figure 6. Electron density maps for pristine (a) and Ag-decorated epitaxial graphene (b, c), showing the effect of extremely thin silver film $(2 \mathrm{~nm}$ and $5 \mathrm{~nm}$ ) on electronic properties of 
graphene. The bottom panel represents the corresponding histograms fitted with a Gaussian function, mean values of the carrier density and standard deviations.
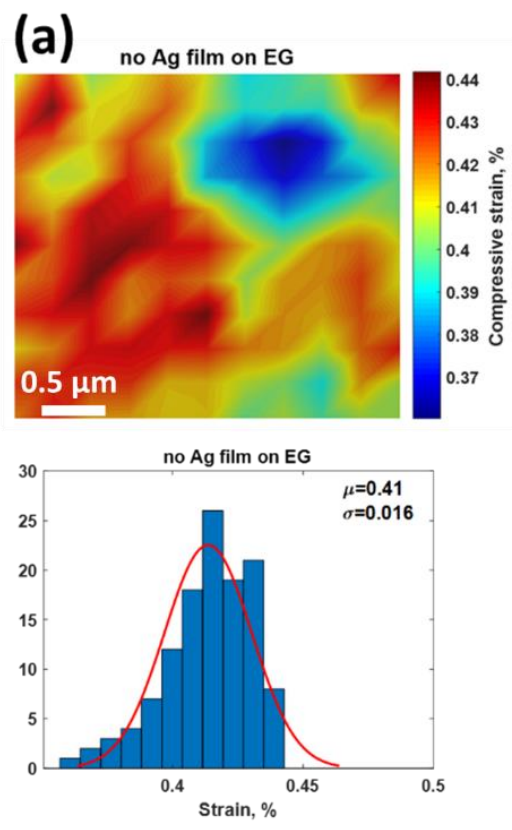
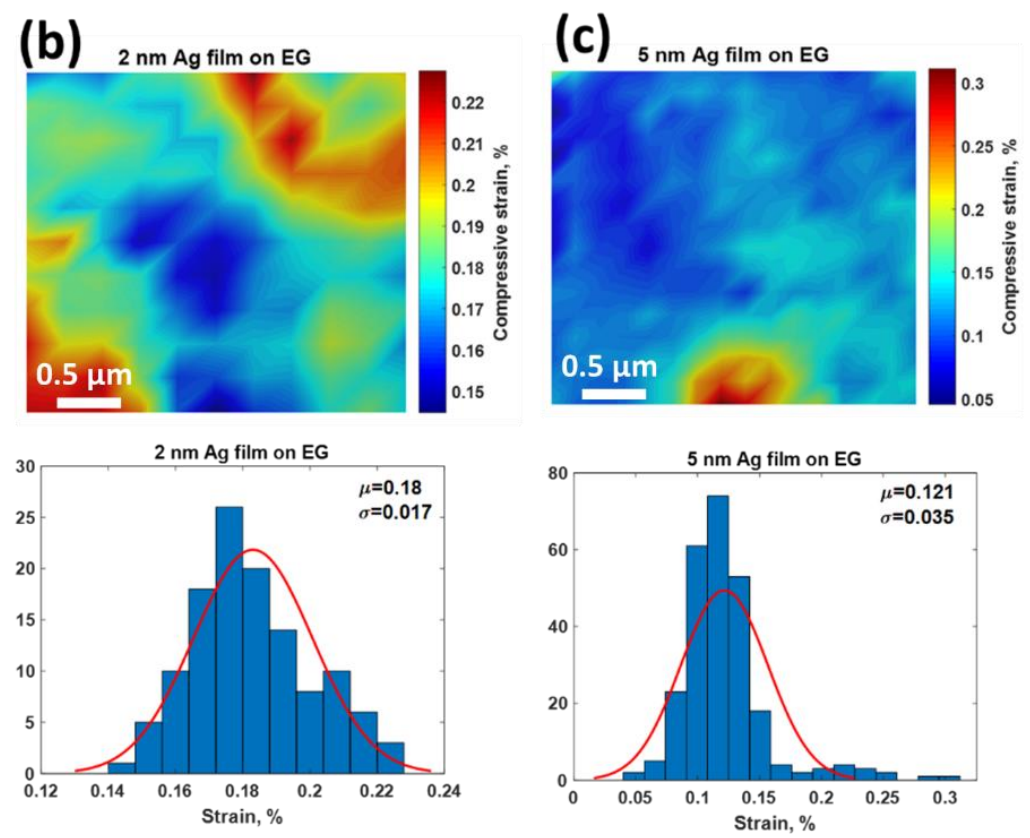

Figure 7. Compressive strain maps for pristine (a) and Ag-decorated epitaxial graphene (b, c), showing the effect of thin Ag film ( $2 \mathrm{~nm}$ and $5 \mathrm{~nm})$ on electronic properties of graphene. The bottom panel represents the corresponding histograms fitted with a Gaussian function, mean values of the compressive strain and standard deviations.

\section{Conclusions}

Large-area uniform electron doping of epitaxial graphene $\left(0.95-1.36 \times 10^{13} \mathrm{~cm}^{-2}\right)$ is achieved by room temperature deposition of extremely thin Ag nano-island films as dopant source. In-depth Raman mapping analysis followed by statistical analysis has been performed to extract the critical information on Ag-induced doping of epitaxial graphene depending on thickness of $\mathrm{Ag}$ nano-film. Epitaxial graphene after decoration with very thin Ag films of $<5 \mathrm{~nm}$ exhibits comparatively narrow red-shifted $2 D$ peak with quite small $2 D / G$ amplitude ratio, suggesting that the successful $n$-doping occurs due to electron transfer from Ag to epitaxial graphene. Thicker Ag films (10 and $15 \mathrm{~nm}$ ) are found to be inappropriate doping sources due to the presence of large strain and doping fluctuations as well as generation of structural in-plane and out-of-plane defects. Interestingly, increase in defect density in all Ag-decorated epitaxial graphene samples causes the unexpected increase the $2 D / G$ amplitude, which can be explained by both inequivalent increase of peak amplitude due to SERS effect and screening effect originating from accommodation of charged impurities at the graphene surface. By constructing the compressive strain and electron doping maps, we revealed the simultaneous reduction of 
the compressive strain and increase in carried density. This observation can be attributed to the partial strain relaxation through a local enhancement of the carrier density. Our results gain deep insights into the Ag effect onto electronic and vibrational properties of epitaxial graphene, and, therefore, can be further utilized to reach better control on doping efficiency.

\section{Acknowledgments}

The authors would like to thank financial support via VR grant 2018-04962, Project \# 302791 and SSF grants (SSF GMT14-0077, SSF RMA15-0024). IS acknowledges also the support from Ångpanneföreningens Forskningsstiftelse (Grant 16-541). KS acknowledges financial support from Linköping University (“LiU Career Contract, Dnr-LiU-2015-01510, 2015-2020”) and the Swedish research council (contract VR-2015-04630). KS and NP acknowledge financial support from the Olle Engkvist foundation (contract SOEB 190-312) and the WennerGren foundations (contract UPD2018-0071).

\section{References}

[1] Shtepliuk, I., Khranovskyy, V., Yakimova, R., 2016. Combining graphene with silicon carbide: synthesis and properties - a review. Semicond. Sci. Technol. 31, 113004. https://doi.org/10.1088/0268-1242/31/11/113004.

[2] Yazdi, G.R., Iakimov, T., Yakimova, R., 2016. Epitaxial Graphene on SiC: A Review of Growth and Characterization. Crystals. 6, 53. https://doi.org/10.3390/cryst6050053.

[3] N. Mishra, J. Boeckl, N. Motta, F. Iacopi, Graphene growth on silicon carbide: A review. Phys. Status Solidi A. 213 (2016) 2277-2289. https://doi.org/10.1002/pssa.201600091.

[4] Kruskopf, M., Pierz, K., Wundrack, S., Stosch, R., Dziomba, T., Kalmbach, C.-C., Müller, A., Baringhaus, J., Tegenkamp, C., Ahlers, F.J., 2015. Epitaxial graphene on SiC: modification of structural and electron transport properties by substrate pretreatment. J. Phys.: Condens. Matter. 27, 185303. https://doi.org/10.1088/0953-8984/27/18/185303.

[5] Riedl, C., Coletti C., Starke, U., 2010. Structural and electronic properties of epitaxial graphene on $\operatorname{SiC}\left(\begin{array}{lll}0 & 0 & 0\end{array}\right)$ : a review of growth, characterization, transfer doping and hydrogen intercalation, J. Phys. D: Appl. Phys. 43, 374009. https://doi.org/10.1088/00223727/43/37/374009.

[6] Shtepliuk, I., Iakimov, T., Khranovskyy, V., Eriksson, J., Giannazzo, F., Yakimova, R., 2017. Role of the Potential Barrier in the Electrical Performance of the Graphene/SiC Interface. Crystals. 7, 162. https://doi.org/10.3390/cryst7060162.

[7] Kruskopf, M., Pakdehi, D.M., Pierz, K., Wundrack, S,. Stosch, R., Dziomba, T., Götz, M., Baringhaus, J., Aprojanz, J., Tegenkamp, C., 2016. Comeback of epitaxial graphene for 
electronics: large-area growth of bilayer-free graphene on SiC. 2D Mater. 3, 041002. https://doi.org/10.1088/2053-1583/3/4/041002.

[8] Santos, C.N., Joucken, F., Meneses, D.D.S., Echegut, P., Campos-Delgado, J., Louette, P., Raskin, J.-P., Hackens, B., 2016. Terahertz and mid-infrared reflectance of epitaxial graphene, Sci Rep. 6, 24301. https://doi.org/10.1038/srep24301.

[9] Santangelo, M.F., Shtepliuk, I., Filippini, D., Puglisi, D., Vagin, M., Yakimova, R., Eriksson, J, 2019. Epitaxial Graphene Sensors Combined with 3D-Printed Microfluidic Chip for Heavy Metals Detection. Sensors. 19, 2393. https://doi.org/10.3390/s19102393.

[10] M.F. Santangelo, I. Shtepliuk, D. Filippini, I.G. Ivanov, R. Yakimova, J. Eriksson, Realtime sensing of lead with epitaxial graphene-integrated microfluidic devices, Sens. Actuators B. 288 (2019) 425-431. https://doi.org/10.1016/j.snb.2019.03.021.

[11] Shtepliuk I., Yakimova R., 2019. Interaction of epitaxial graphene with heavy metals: towards novel sensing platform. Nanotechnol. 30, 294002. https://doi.org/10.1088/13616528/ab1546.

[12] Shtepliuk, I., Santangelo, M.F., Vagin, M., Ivanov, I.G., Khranovskyy, V., Iakimov, T., Eriksson, J., Yakimova, R., 2018. Understanding Graphene Response to Neutral and Charged Lead Species: Theory and Experiment. Materials. 11, 2059. https://doi.org/10.3390/ma11102059.

[13] K. He, Z. Zeng, A. Chen, G. Zeng, R. Xiao, P. Xu Z. Huang, J. Shi, L. Hu, G. Chen, 2018. Advancement of Ag-Graphene Based Nanocomposites: An Overview of Synthesis and Its Applications. Small. 14, 1800871. https://doi.org/10.1002/smll.201800871.

[14] S.S. Sukumarana, C.R. Rekha, A.N. Resmi, K.B. Jinesh, K.G. Gopchandran, Raman and scanning tunneling spectroscopic investigations on graphene-silver nanocomposites, J. Sci.: Adv. Mater. Devices, 3 (2018) 353-358. https://doi.org/10.1016/j.jsamd.2018.06.003.

[15] Amft, M., Lebègue, S., Eriksson, O., Skorodumova, N.V., 2011. Adsorption of Cu, Ag, and $\mathrm{Au}$ atoms on graphene including van der Waals interactions. J. Phys.: Condens. Matter. 23, 395001. https://doi.org/10.1088/0953-8984/23/39/395001.

[16] Hsu, C.-H., Lin, W.-H., Ozolins, V., Chuang, F.-C., 2012. Electronic structures of an epitaxial graphene monolayer on $\mathrm{SiC}(0001)$ after metal intercalation (metal $=\mathrm{Al}, \mathrm{Ag}, \mathrm{Au}, \mathrm{Pt}$, and Pd): A first-principles study. Appl. Phys. Lett. 100, 063115. https://doi.org/10.1063/1.3682303.

[17] S. Chadhari, A.R. Graves, M.V. Cain, C.D. Stinespring, "Graphene-based composite sensors for energy applications," Proc. SPIE 9836, Micro- and Nanotechnology Sensors, Systems, and Applications VIII, 98360G (17 May 2016); 
[18] M. Homa, N. Sobczak, J.J.Sobczak, A. Kudyba, G. Bruzda, R. Nowak, D. Giuranno, K. Pietrzak, M. Chmielewski, Interaction Between Liquid Silver and Graphene-Coated SiC Substrate, J. Mater. Eng. Perform. 27 (2018) 4140-4149. https://doi.org/10.1007/s11665-0183503-7.

[19] X. Liu, T. Hu, Y. Miao, D. Ma, Z. Yang, F. Ma, K. Xu, P.K. Chu, Substitutional doping of $\mathrm{Ag}$ into epitaxial graphene on $6 \mathrm{H}-\mathrm{SiC}$ substrates during thermal decomposition, Carbon. 104 (2016) 233-240. https://doi.org/10.1016/j.carbon.2016.04.007.

[20] Y. Sekine, H. Hibino, K. Oguri, T. Akazaki, H. Kageshima, M. Nagase, K.-I. Sasaki, H. Yamaguchi, Surface-enhanced raman scattering of graphene on SiC, NTT Technical Review, $11(2013) 8$.

[21] Liu, X., Fang, Q., Hu, T., Ma, D., Zhang, X., Liu, S., Ma, F., Xu, K. 2018. Thickness dependent Raman spectra and interfacial interaction between $\mathrm{Ag}$ and epitaxial graphene on $6 \mathrm{H}-$ SiC(0001). Phys.Chem.Chem.Phys., 20, 5964. https://doi.org/10.1039/c7cp07338k.

[22] Hu, T., Liu, X., Ma, D., Wei, R., Xu, K., Ma, F., 2018. Formation of Micro- and NanoTrenches on Epitaxial Graphene. Appl. Sci. 8, 2518. https://doi.org/10.3390/app8122518.

[23] Verhagen, T.G.A., Drogowska, K., Kalbac, M., Vejpravova, J., 2015. Temperature induced strain and doping in monolayer and bilayer isotopically labeled graphene. Phys. Rev. B. 92, 125437, https://doi.org/10.1103/PhysRevB.92.125437.

[24] A. Das, S. Pisana, B. Chakraborty, S. Piscanec, S.K. Saha, U.V. Waghmare, K.S. Novoselov, H.R. Krishnamurthy, A.K. Geim, A.C. Ferrari \& A. K. Sood, Monitoring dopants by Raman scattering in an electrochemically top-gated graphene transistor. Nat. Nanotechnol. 3 (2008) 210-215. https://doi.org/10.1038/nnano.2008.67.

[25] Lee, J.E., Ahn, G., Shim, J., Lee, Y.S., Ryu, S., 2012. Optical separation of mechanical strain from charge doping in graphene. Nat. Commun. 3, 1024. https://doi.org/10.1038/ncomms2022 .

[26] Mueller, N.S., Heeg, S., Alvarez, M.P., Kusch, P., Wasserroth, S., Clark, N., Schedin, F., Parthenios, J., Papagelis, K., Galiotis, C., 2018. Evaluating arbitrary strain configurations and doping in graphene with Raman spectroscopy. 2D Mater. 5, 015016. https://doi.org/10.1088/2053-1583/aa90b3.

[27] I. Shtepliuk, I.G. Ivanov, T. Iakimov, R. Yakimova, A. Kakanakova-Georgieva, P. Fiorenza, F. Giannazzo, Raman probing of hydrogen-intercalated graphene on Si-face 4H-SiC. Mater. Sci. Semicond. Proces. 96 (2019) 145-152. https://doi.org/10.1016/j.mssp.2019.02.039. [28] Giannazzo, F., Shtepliuk, I., Ivanov, I.G., Iakimov, T., Kakanakova-Georgieva, A., Schilirò, E., Fiorenza, P., Yakimova, R., 2019. Probing the uniformity of hydrogen intercalation 
in quasi-free-standing epitaxial graphene on $\mathrm{SiC}$ by micro-Raman mapping and conductive atomic force microscopy. Nanotechnology. 30, 284003. https://doi.org/10.1088/13616528/ab134e.

[29] Yakimova, R.; Iakimov, T.; Syväjärvi, M. Process for Growth of Graphene. U.S. Patent US9150417B2, 6 October 2015.

[30] I.G. Ivanov, J. Hassan, T. Iakimov, A.A. Zakharov, R. Yakimova, E. Janzén, Layernumber determination in graphene on SiC by reflectance mapping. Carbon. 77 (2014) 492-500. https://doi.org/10.1016/j.carbon.2014.05.054.

[31] V. Elofsson, B. Lü, D. Magnfält, E.P. Münger, and K. Sarakinos, Unravelling the physical mechanisms that determine microstructural evolution in ultra-thin Volmer-Weber films, J. Appl. Phys. 116 (2014) 044302. https://doi.org/10.1063/1.4890522

[32] A. Jamnig, D.S. Sangiovanni, G. Abadias, and K. Sarakinos, Atomic-scale diffusion rates during growth of thin metal films on weakly-interacting substrates, Sci. Rep. 9 (2019) 6640. https://doi.org/10.1038/s41598-019-43107-8

[33] K. Sarakinos, A review on morphological evolution of thin metal films on weaklyinteracting substrates, Thin Solid Films, in press (2019), https://doi.org/10.1016/j.tsf.2019.05.031

[34] Rohrl, J., Hundhausen, M., Emtsev, K., Seyller, T., Graupner, R., Ley, L., 2008. Appl. Phys. Lett. 92, 201918. https://doi.org/10.1063/1.2929746.

[35] Fromm, F., Oliveira Jr, M.H., Molina-Sanchez ', A., Hundhausen, M., Lopes, J. M. J., Riechert, H., Wirtz, L., Seyller, T., 2013. Contribution of the buffer layer to the Raman spectrum of epitaxial graphene on SiC(0001). New J. Phys. 15, 043031. https://doi.org/10.1088/1367-2630/15/4/043031.

[36] R. Saito, M. Hofmann, G. Dresselhaus, A. Jorio, M. S. Dresselhaus, Adv. Phys. 30 (2011) 413-550. https://doi.org/10.1080/00018732.2011.582251.

[37] Saito, R., Jorio, A., Souza Filho, A. G., Dresselhaus, G., Dresselhaus, M. S., Pimenta, M., 2002. A. Phys. Rev. Lett. 88, 027401. https://doi.org/10.1103/PhysRevLett.88.027401.

[38] Zheng, X., Chen, W., Wang, G., Yu, Y., Qin, S., Fang, J., Wang, F., Zhang, X.-A., 2015. The Raman redshift of graphene impacted by gold nanoparticles. AIP Adv. 5, 057133. https://doi.org/10.1063/1.4921316.

[39] F. Schedin, E. Lidorikis, A. Lombardo, V.G. Kravets, A.K. Geim, A.N. Grigorenko, K.S. Novoselov, Andrea C. Ferrari, Surface-Enhanced Raman Spectroscopy of Graphene. ACS Nano. 4 (2010) 5617-5626. https://doi.org/10.1021/nn1010842. 
[40] S. Sun, P. Wu, Competitive surface-enhanced Raman scattering effects in noble metal nanoparticle-decorated graphene sheets, Phys. Chem. Chem. Phys. 13 (2011) 21116-21120. https://doi.org/10.1039/C1CP22727K.

[41] S. Berciaud, X. Li, H. Htoon, L.E. Brus, S.K. Doorn, T.F. Heinz, Intrinsic line shape of the Raman-2D mode in freestanding graphene monolayers, Nano Lett. 13 (2013) 3517-3523. https://doi.org/10.1021/n1400917e.

[42] J.E. Lee, G. Ahn, J. Shim, Y.S. Lee, S. Ryu, 2012. Optical separation of mechanical strain from charge doping in graphene. Nat. Commun. 3, 1024 https://doi.org/10.1038/ncomms2022. [43] Ni, Z.H., Chen, W., Fan, X.F., Kuo, J.L., Yu, T., Wee, A.T.S., Shen, Z.X., 2008. Raman spectroscopy of epitaxial graphene on a SiC substrate. Phys. Rev. B. 77, 115416. https://doi.org/10.1103/PhysRevB.77.115416.

[44] Fromm, F., Wehrfritz, P., Hundhausen, M., Seyller, Th., 2013. Looking behind the scenes: Raman spectroscopy of top-gated epitaxial graphene through the substrate. New J. Phys. 15, 113006. https://doi.org/10.1088/1367-2630/15/11/113006.

[45] Banszerus, L., Janssen, H., Otto, M., Epping, A., Taniguchi, T., Watanabe, K., Beschoten, B., Neumaier, D., Stampfer, C., 2017. Identifying suitable substrates for high-quality graphene$\begin{array}{lllll}\text { based heterostructures. } & \text { 2D } & \text { Mater. } & 4, & 025030 .\end{array}$ https://doi.org/10.1088/2053-1583/aa5b0f.

[46] Varykhalov A., Scholz, M. R., Kim, Timur K., Rader, O., 2010. Effect of noble-metal contacts on doping and band gap of graphene. Phys. Rev. B. 82, 121101(R). https://doi.org/10.1103/PhysRevB.82.121101.

[47] Papagno, M., Moras, P., Sheverdyaeva, P.M., Doppler, J., Garhofer, A., Mittendorfer, F., Redinger, J., Carbone, C., 2013. Hybridization of graphene and a Ag monolayer supported on Re(0001). Phys. Rev. B. 88, 235430. https://doi.org/10.1103/PhysRevB.88.235430.

[48] J. Lee, K.S. Novoselov, H.S. Shin, Interaction between Metal and Graphene: Dependence on the Layer Number of Graphene, ACS Nano. 5 (2011) 608-612. https://doi.org/10.1021/nn103004c.

[49] Venezuela, P., Lazzeri, M., Mauri, F., 2011. Theory of double-resonant Raman spectra in graphene: intensity and line shape of defect-induced and two-phonon bands. Phys Rev B. 84, 035433. https://doi.org/10.1103/PhysRevB.84.035433.

[50] D. M. Basko, S. Piscanec, and A. C. Ferrari, Electron-electron interactions and doping dependence of the two-phonon Raman intensity in graphene, Phys. Rev. B 80 (2009) 165413. [51] J. M. Urban, P. Dąbrowski, J. Binder, M. Kopciuszyński, A. Wysmołek, Z. Klusek, M. 
Jałochowski, W. Strupiński, and J. M. Baranowski, J. Appl. Phys. 115, 233504 (2014); https://doi.org/10.1063/1.4884015

[52] Schmidt, D.A., Ohta, T., Beechem, T.E., 2011. Strain and charge carrier coupling in epitaxial graphene. Phys. Rev. B. 84, 235422. https://doi.org/10.1103/PhysRevB.84.235422. 